\title{
La excepción de convenio arbitral en la Ley Peruana de Arbitraje y la Convención de Nueva York
}

\author{
Nicolás Serván Eyzaguirre* \\ Daichi Yano Tsuha*
}

Resumen.- La Ley Peruana de Arbitraje y la Convención de Nueva York regulan la excepción de convenio arbitral de forma distinta. Ambas con criterios distintos para remitir a las partes a arbitraje. En este contexto, la Ley Peruana resulta ser más favorable al arbitraje pues establece que el análisis del juez debe ser prima facie. Por lo tanto, en virtud al principio de máxima eficacia, el juez debe resolver la excepción sustentado en la Ley Peruana.

Abstract.- The Peruvian Arbitration Law and the New York Convention regulate the objection as to that court's lack of jurisdiction on the basis of the existence of the arbitration agreement differently from each other. Both have different criteria in order to refer the parties to arbitration. In this sense, the Peruvian Arbitration Law appears to be the most favorable to arbitrate since it establishes that the analysis made by the judge must be prima facie. Therefore, according to the principle of maximum effectiveness, the judge must solve the objection based on the Peruvian Arbitration Law.

Estudiante de sexto ciclo de la carrera de Derecho en la Universidad del Pacífico. Sub-director de la Comisión de Edición de Forseti - Revista de Derecho.

* Estudiante de sexto ciclo de la carrera de Derecho en la Universidad del Pacífico. Director de la Comisión de Finanzas de Forseti - Revista de Derecho. 


\section{Introducción}

Desde que existe un convenio arbitral, puede surgir una patología. Así, cuando los pactos entre las partes no son lo suficientemente precisos y claros o algún supuesto de hecho afecta su compromiso a arbitrar, nos encontramos ante una patología del convenio arbitral. Es pacífica la doctrina al afirmar que el convenio arbitral obliga a las partes a resolver las controversias que se encuentren dentro de su alcance en la vía arbitral, pero ¿hasta qué punto llega esa obligación? El límite podrá encontrarse, en determinados casos, en la imposibilidad de ejecutar la voluntad de las partes. Inclusive, aun cuando no sea posible respetar estrictamente su voluntad, habrá algunos supuestos en donde las partes seguirán obligadas por el convenio arbitral pues la patología es subsanable y no le devolverá la competencia al "juez natural".

De inmediato surge la interrogante sobre quién debe determinar si el convenio arbitral es patológico y si, tal como está redactado, no permite que las partes resuelvan sus controversias en esa vía. $\mathrm{O}$, en caso contrario, sigue obligando a las partes. Lo cierto es que la respuesta cambiará en la medida ante quién se alegue la supuesta patología: por ejemplo, si se realiza en el marco de un proceso judicial, nos encontraremos frente a una excepción de convenio arbitral. En este contexto, específicamente analizaremos si, en el marco de un arbitraje internacional, la Ley Peruana de Arbitraje establece parámetros distintos a los establecidos por la Convención de Nueva York, a fin de remitir a las partes a arbitraje, o si estipula las mismas condiciones para denegar la excepción de incompetencia por convenio arbitral. En concreto, buscaremos determinar si la excepción del artículo 16 de la Ley Peruana de Arbitraje es más favorable que el artículo 2.3 de la Convención de Nueva York.

Asimismo, buscaremos determinar si el juez debe hacer un análisis a profundidad, o prima facie, sobre la patología invocada para remitir a las partes a arbitraje. Si la controversia finalmente llega donde los árbitros, ¿estos deberán hacer un análisis en los mismos términos que el juez? ¿Qué sucedería si, contrario a lo resuelto por el juez, el tribunal arbitral considerara que la cláusula patológica no es salvable?

En este artículo abordaremos, en primer lugar, las obligaciones que surgen de un convenio arbitral. Luego, analizaremos la naturaleza de la excepción de convenio arbitral y específicamente las excepciones reguladas en la Convención de Nueva York y la Ley Peruana de Arbitraje. Finalmente, realizaremos un contraste entre ambas excepciones, a fin de determinar si una 
es más favorable que la otra, y cómo se debe proceder en caso de concurrencia de aplicabilidad. ¿Será que la Ley Peruana es más favorable?

\section{Las obligaciones del convenio arbitral}

El artículo 13 del Decreto Legislativo No 1071 (en adelante, la "Ley Peruana de Arbitraje") establece que "el convenio arbitral es un acuerdo por el que las partes deciden someter a arbitraje todas las controversias o ciertas controversias que hayan surgido o puedan surgir entre ellas respecto de una determinada relación jurídica contractual o de otra naturaleza". Como bien señala González de Cossío "el acuerdo arbitral es un contrato por virtud del cual dos o más partes acuerdan que una controversia, ya sea presente o futura, se resuelva mediante arbitraje" $\mathrm{e}^{1}$. Por ese compromiso a arbitrar, las partes tienen la obligación de resolver sus controversias en arbitraje, y no en otra vía, como podría ser la del Poder Judicial.

Gary Born señala que el convenio arbitral genera dos obligaciones para las partes contratantes: una positiva y otra negativa. Expresamente sostiene que "un convenio de arbitraje internacional válido produce efectos legales importantes para las partes, así como para las cortes nacionales y los árbitros. Esos efectos de los convenios arbitrales son positivos y negativos: los efectos positivos incluyen la obligación de participar y cooperar de buena fe en el procedimiento arbitral de acuerdo con el convenio arbitral de las partes; mientras que los efectos negativos incluyen la obligación de no buscar la resolución de disputa sujetas a arbitraje en las cortes nacionales o jurisdicciones similares" 2 .

En nuestro medio, Carlos Soto ha sido enfático al afirmar lo siguiente:

“Una vez celebrado un convenio arbitral las partes contratantes están obligadas a:

- Someter sus controversias a un proceso arbitral (obligación de hacer).

GONZÁLEZ DE COSSÍO, Francisco. “Arbitraje” 2004. p.56.

2 Traducción libre de: A valid international arbitration agreement produces important legal effects for its parties, as well as for national courts and arbitrators. (1) These effects of arbitration agreements are both positive and negative: the positive effects include the obligation to participate and cooperate in good faith in the arbitration of disputes pursuant to the parties' arbitration agreement, while the negative effects include the obligation not to pursue the resolution of disputes that are subject to arbitration in national courts or similar legal forum.

BORN, Gary. "International Commercial Arbitration". Segunda edición. Kluwer Law International; Kluwer Law International, 2014, p. 1253. 
- Cumplir con los mandatos impuestos en el laudo arbitral (obligación de hacer).

- No acudir a los tribunales judiciales para resolver sus controversias, pues al celebrar el convenio arbitral han renunciado a la competencia del Poder Judicial (obligación de no hacer)"3.

Si bien las obligaciones del convenio arbitral son claras, ¿qué sucede en el caso en el que una parte del convenio arbitral considera que este ya no es ejecutable porque, por ejemplo, la institución arbitral que designaron desapareció? ¿Deberá demandar ante el Poder Judicial? ¿Seguirá estando obligada por el convenio arbitral? ¿Un juez deberá determinar si el convenio sigue obligando a las partes? ¿O lo deberán hacer los árbitros?

Consideramos que la parte demandante debería hacer una previa evaluación del convenio arbitral, a fin de determinar la vía idónea para presentar su demanda. Si considera que es posible ejecutar el convenio, no existirá mayor problema al respecto; sin embargo, si considera que este es de imposible ejecución y demanda ante el Poder Judicial, lo más probable es que nos encontremos frente a una excepción de convenio del demandado. Este último alegará que se ha vulnerado la obligación negativa que se desprende del convenio arbitral, y el juez debe remitir a las partes a arbitraje.

\section{La excepción de convenio arbitral}

En el presente acápite pretendemos comparar la excepción de convenio arbitral regulada en el artículo 16 de la Ley Peruana de Arbitraje con la establecida en el artículo 2.3 de la Convención de Nueva York. Si bien a primeras luces, la inquietud planteada parece meramente referencial y académica, la misma si presenta implicancias prácticas en la realidad, como veremos a continuación.

\section{i. La naturaleza de la excepción de convenio arbitral.}

En un proceso judicial, el demandado tiene dos posibles defensas que plantear. Así, puede plantear tanto una defensa de forma como de fondo. El tema que nos ocupa -es decir, la excepción- es típicamente el primer caso. Al respecto, el profesor Juan Monroy señala que "la defensa de forma o excepción consiste, entre otras cosas, en alegar la existencia de una relación jurídico procesal defectuosa, quien intente una excepción lo estará haciendo - en una de sus dos posibilidades - es afirmar la ausencia o presencia defectuosa de uno o más

SOTO, Carlos y BULLARD, Alfredo. "Comentarios a la ley peruana de arbitraje". Lima: Instituto Peruano de Arbitraje Comercial y Arbitraje de Inversiones, 2011, p. 155. 
presupuestos procesales" 4 . En resumidas cuentas, una excepción permite cuestionar desde la competencia y el interés para obrar, hasta la cosa juzgada y la prescripción extintiva 5 .

Por su parte, Adrián Simons, sobre la referida excepción, señala que "es considerada como una de naturaleza perentoria simple, porque extingue el proceso judicial sin afectar la pretensión objeto de discusión. En este caso, [...] el defecto procesal que se debería advertir al momento de su postulación es la ausencia de interés procesal (interés para obrar) o sea una condición de la acción, ya que ese estado de necesidad ha sido dirigido de manera errónea (juez en lugar de árbitro). Por ende, no considero que el impedimento procesal esté referido a la falta de competencia del juez o, como erróneamente se menciona, también a la "falta de jurisdicción""6.

La otra posición sugiere que el juez no sería competente por lo que la excepción de convenio arbitral sería puramente una de incompetencia. A dicha tesis se adscriben autores como Roque Caivano, quien afirma que "si alguna de las partes iniciara una acción judicial relacionada con dichas cuestiones, la otra parte podrá plantear la incompetencia del tribunal judicial, sobre la base de que su jurisdicción ha sido renunciada". Su posición se hace más clara cuando sostiene que "el artículo 349 dispone que, para dar curso a la excepción de incompetencia, fundada en haberse fijado de común acuerdo por las partes el juez competente'"7.

Si bien la mayoría se inclina entre las posturas descritas, consideramos que la excepción de convenio arbitral no es ni una excepción de incompetencia, ni una excepción de falta de interés para obrar, pues hasta el propio Código Procesal Civil las ha regulado de manera separada. La finalidad de la

4 MONROY GÁlVEZ, Juan. “Las excepciones en el código procesal civil peruano". Lima: THĒMIS-Revista de Derecho, 2015 p. 122.

5 Del artículo 446 del Código Procesal Civil se desprende que existen hasta 13 tipos de excepciones expresamente reguladas: incompetencia, incapacidad del demandante o de su representante, representación defectuosa o insuficiente del demandante o del demandado, oscuridad o ambigüedad en el modo de proponer la demanda, falta de agotamiento de la vía administrativa, falta de legitimidad para obrar del demandante o demandado, litispendencia, cosa juzgada, desistimiento de la pretensión, conclusión del proceso por conclusión o transacción, caducidad, prescripción extintiva y convenio arbitral.

6 SIMONS, Adrian en SOTO, Carlos y BULLARD, Alfredo. “Comentarios a la ley peruana de arbitraje". Lima: Instituto Peruano de Arbitraje Comercial y Arbitraje de Inversiones, 2011, pp. 264-265.

7 CAIVANO, Roque. (2012). “La Cláusula Arbitral y la Cesión del Contrato que la Contiene”. Revista de Derecho Privado, p. 6.

Recuperado el 21 de octubre de 2017 de: https:// revistascolaboracion.juridicas.unam.mx/index.php/derecho-privadons/article/viewFile/7246/6525 
excepción de convenio arbitral es impedir que el juez analice el fondo de la controversia, pues las partes celebraron un contrato en el que se obligaban a resolver sus controversias mediante arbitraje y renunciaban a la jurisdicción ordinaria.

Como comentario final, nos remitimos a lo expresado por Roger Rubio, quien sostiene que "un antecedente directo del Competence-Competence en su vertiente negativa puede encontrarse en el artículo II.3 de la Convención sobre el Reconocimiento y Ejecución de las Sentencias Arbitrales Extranjeras armado en Nueva York en 1958"8. Es decir, la excepción de convenio arbitral - regulada en el artículo II.3 de la Convención de Nueva York - es una manifestación del Competence-Competence en su vertiente negativa, pues este principio permite a los árbitros pronunciarse sobre la validez del convenio arbitral ${ }^{9}$. Justamente, lo que analizaremos a profundidad más adelante es si los jueces deben determinar la validez absoluta del convenio arbitral, o si esta es una labor que le compete a los árbitros.

\section{ii. Ámbito de aplicación}

El inciso 2 del artículo 1 de la Ley Peruana de Arbitraje, estipula lo siguiente: “las normas contenidas en los numerales 1, 2, 3, 5 y 6 del artículo 8, en los

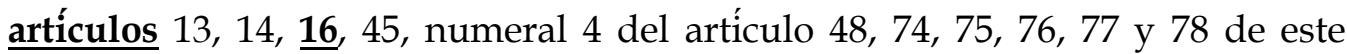
Decreto Legislativo, se aplicarán aun cuando el lugar del arbitraje se halle fuera del Perú" (el énfasis es nuestro). Es decir, la excepción del artículo 16 de la Ley Peruana de Arbitraje se puede aplicar también cuando las partes han pactado un arbitraje que se llevará a cabo en el extranjero.

Por su parte, la Convención de Nueva York regula su ámbito de aplicación en el artículo I; sin embargo, no se pronuncia sobre la aplicación del artículo II.3, que contiene la excepción de convenio arbitral. Al respecto, Van Den Berg señala lo siguiente:

“En el caso del convenio que estipula un arbitraje en otro Estado, el artículo I de la Convención puede ser aplicado por analogía. Así como la Convención aplica para ejecución de un laudo hecho en otro Estado, ella puede aplicar para la ejecución de un convenio arbitral que establece el arbitraje en otro Estado. Un examen de las decisiones de las Cortes que aplican el artículo II.3, revela que la mayoría de ellas implica un convenio que provee arbitraje en otro Estado. La aplicación de la Convención a esta categoría de

8 RUBIO, Roger. “El principio Competence-Competence en la nueva Ley Peruana de Arbitraje". En Lima Arbitration. No 4, 2011, p. 102.

9 Idem. 
convenios arbitrales parece ser tan evidente que ningún tribunal consigue explicar por qué aplica la Convención"10.

Debemos señalar que algunas Cortes han interpretado que la Convención de Nueva York también aplica para convenios arbitrales que estipulan un arbitraje comercial internacional en el propio Estado ${ }^{11}$, aunque ello no se desprenda de una interpretación sistemática con el artículo I.

Queremos dejar en claro que, en determinados supuestos, es posible que se aplique conjuntamente la excepción de convenio arbitral regulada en la Ley Peruana de Arbitraje; así como la contenida en la Convención de Nueva York. En ese supuesto, será de aplicación el análisis que realizaremos posteriormente.

\section{iii. La excepción de convenio arbitral en la Convención de Nueva York}

El artículo II.3 reza de la siguiente manera:

“El tribunal de uno de los Estados contratantes al que se someta un litigio respecto del cual las partes hayan concluído un acuerdo en el sentido del presente artículo, remitirá a las partes al arbitraje, a instancia de una de ellas, a menos que compruebe que dicho acuerdo es nulo, ineficaz o inaplicable" . (el énfasis es nuestro)

El propio inciso nos remite a lo regulado en el inciso 1 del artículo II, que establece los requisitos del convenio arbitral para que sea reconocido por parte de los Estados contratantes de la Convención. Por lo tanto, ante la presencia de un convenio arbitral, el Tribunal Judicial deberá remitir a las partes a arbitraje. Van den Berg señala que por "remisión" se entiende que existe un mandato hacia los jueces. En ese sentido, "el carácter mandatorio de la remisión por parte de una Corte a Arbitraje en virtud al artículo II.3 es una regla internacional uniforme. Esta sobrepasa la ley interna que establece que las

10 Traducción libre de: "In the case of an agreement providing for arbitration in another State, Article I of the Convention could be applied by analogy. As the Convention applies to the enforcement of an award made in another State, it could apply to the enforcement of an agreement providing for arbitration in another State.

An examination of the court decisions in which an application under Article II(3) was made, reveals that the majority of them involved an agreement providing for arbitration in another State. The application of the Convention to this category of arbitration agreements appears to be so self-evident that almost no court gave an explanation why it applied the Convention". VAN DEN BERG, J. Albert. "The New York Arbitration Convention of 1958". Kluwer International Arbitration, 1989, p. 57.

11 Idem. 
cortes tienen un poder discrecional si debe o no permanecer la acción judicial en violación a un convenio arbitral"12.

La única condición que contempla la Convención de Nueva York, para que los jueces remitan a las partes al arbitraje, es que el convenio arbitral sea válido, eficaz y aplicable. De lo contrario podrían denegar la excepción de convenio arbitral y analizar el fondo de la controversia. Sin embargo, de la redacción de la norma no resulta posible determinar si el análisis de la nulidad, eficacia y aplicabilidad debe ser completo o prima facie. En el "Case Law" de la Ley Modelo Uncitral, que regula la excepción de convenio arbitral en los mismos términos que la Convención de Nueva York se señala lo siguiente:

“En diversas jurisdicciones incluyendo Croacia, España, México, Australia, Uganda y Kenia, los casos muestran que las Cortes [...] han adoptado la visión de que se requiere una revisión completa. Aspectos como validez, eficacia y aplicabilidad deben por consiguiente ser analizados en su totalidad, y las decisiones relacionadas por lo tanto deben ser definitivas.

[...] Sin embargo, Cortes en otras jurisdicciones han preferido aplicar un estándar prima facie bajo el sustento que los árbitros están empoderados para determinar su propia jurisdicción"13.

Si bien no existe un criterio uniforme y claro sobre cuál es el tipo de análisis que debe efectuar el juez, lo innegable es que debe analizar la nulidad, eficacia y aplicabilidad del convenio arbitral. En ese contexto, corresponde pronunciarnos sobre las tres categorías mencionadas.

\section{La nulidad, ineficacia e inaplicabilidad}

\section{i) Convenio arbitral nulo}

12 Traducción libre de: "The mandatory character of the referral by a court to arbitration pursuant to Article II(3) is an internationally uniform rule. It supersedes domestic law which may provide that the court has a discretionary power whether or not to stay a court action brought in violation of an arbitration agreement".

VAN DEN BERG, J. Albert. "The New York Arbitration Convention of 1958". Kluwer International Arbitration, 1989, p. 135.

13 Traducción libre de: "“In several jurisdictions, including Croatia, Spain, Mexico, Australia, Uganda and Kenya, the cases show that courts have either not considered this issue or not considered it in detail, and have adopted the view that a full review would be required. Issues of validity, operativeness, performability and applicability would thus be analysed fully, and decisions relating thereto should be final.204 In Ireland, a court that analysed the issue in detail said to be inclined to favour that standard.

However, courts in other jurisdictions have preferred to apply a prima facie standard on the ground that, as arbitrators are empowered to rule on their own jurisdiction [...]"

UNCITRAL. "Digest of Case Law on the Model Law on International Commercial Arbitration". 2012 , p.44.

Recuperado de: http://www.uncitral.org/pdf/english/clout/MAL-digest-2012-e.pdf 
La nulidad es entendida como la ausencia de cualquiera de los requisitos de validez establecidos por la ley aplicable al convenio arbitral. Así, González de Cossío señala que "se refiere a circunstancias que, bajo el derecho aplicable al acuerdo arbitral, vicia la validez de origen del acuerdo arbitral [...] Debe, por consiguiente, probarse que el acuerdo arbitral mismo está viciado de dolo, mala fe, error" 14 .

Debemos tener en cuenta que aunque la propia Convención no ha definido qué debe entenderse por nulidad, la misma ha establecido un estándar de validez del convenio arbitral en el artículo II.1. Este artículo establece lo siguiente: “cada uno de los Estados Contratantes reconocerá el acuerdo por escrito conforme al cual las partes se obliguen a someter a arbitraje todas las diferencias o ciertas diferencias que hayan surgido o puedan surgir entre ellas respecto a una determinada relación jurídica, contractual o no contractual, concerniente a un asunto que pueda ser resuelto por arbitraje".

$\mathrm{Al}$ respecto debemos tener en cuenta lo señalado por Van den Berg:

“Estas condiciones son: la disputa debe surgir con respecto a una relación legal definida (Art. II (1)), la materia debe ser capaz de resolverse por arbitraje (Art. II (1)), y el acuerdo de arbitraje debe ser por escrito (Art. II (1) y (2)). Por lo tanto, las palabras "null and void" no se aplican a estas condiciones [...] los términos deben ser interpretados como referidos a esos casos donde el convenio arbitral está afectado por alguna invalidez desde el inicio. Por ello, cubriría asuntos como la falta de consentimiento debido a malinterpretaciones, coacción, fraude o influencia indebida"15.

Sin embargo, desde nuestra perspectiva nada impide que las Cortes puedan determinar la invalidez del convenio arbitral si no cumple con los requisitos establecidos en el artículo II.1 y II.2.

\section{ii) Convenio arbitral ineficaz}

La ineficacia de un convenio arbitral se produce porque el mismo no genera efectos jurídicos entre las partes. Así lo señala la Guía para la Interpretación de la Convención de Nueva York en los siguientes términos: “un acuerdo arbitral

14 GONZÁLEZ DE COSSÍO, Francisco. “Arbitraje”. 2004, p 189.

15 Traducción libre de: "The words "null and void" therefore do not apply to these conditions. This is, however, merely a question of system and has no legal consequences. [...] The words may be interpreted as referring to those cases where the arbitration agreement is affected by some invalidity right from the beginning. It would then cover matters such as the lack of consent due to misrepresentation, duress, fraud or undue influence"

VAN DEN BERG, J. Albert. "The New York Arbitration Convention of 1958". Kluwer International Arbitration, 1989, p. 155-156. 
ineficaz para efectos del artículo II.3 es un acuerdo arbitral que en algún momento tuvo validez pero que ha cesado de tener efectos. La excepción de "ineficacia" típicamente incluye casos de renuncia, revocación o terminación del acuerdo arbitral. Similarmente, el acuerdo arbitral debe de considerarse ineficaz si la misma disputa entre las mismas partes ya ha sido decidida por una corte o tribunal arbitral (res judicata o ne bis in idem)"16.

Por su parte, Redfern y Hunter sostienen que "a primera vista, es difícil ver la distinción entre los términos 'ineficaz' e 'inaplicable'. Sin embargo, un convenio arbitral es ineficaz donde este ha dejado de tener efectos legales como resultado, por ejemplo, de un fracaso de las partes para complir con un tiempo límite, o donde las partes han renunciado, o por su conducta implícitamente revocado el convenio arbitral"17.

\section{iii) Convenio arbitral inaplicable}

Los impedimentos físicos a un procedimiento arbitral o hechos sobrevinientes a la celebración del convenio arbitral, dependiendo de las disposiciones particulares de la ley aplicable a la ejecución del convenio arbitral, pueden llevar a la imposibilidad del cumplimiento del convenio. No son ajenos a la realidad los supuestos en los que la mala redacción de las cláusulas arbitrales impidan el inicio del procedimiento arbitral. A estas cláusulas defectuosas, generalmente, se les denomina "patológicas". Estos convenios arbitrales son frecuentemente cuestionados ante las Cortes nacionales, en virtud a las dificultades que tienen para poner en práctica lo establecido en dicho acuerdo. Tales cláusulas deben de interpretarse de acuerdo con la misma ley que rige la formación y validez sustantiva del acuerdo arbitral ${ }^{18}$.

\section{Los convenios arbitrales patológicos}

Mención aparte merece la patología de los convenios arbitrales, pues a pesar de ser un tema afín, es necesario tratarlo por sí mismo. El término "convenios

16 INTERNATIONAL COUNCIL OF COMMERCIAL ARBITRATION. “Guía del ICCA para la interpretación de la Convención de Nueva York de 1958". 2013, p. 53.

Recuperado de:

http://www.oas.org/es/sla/ddi/docs/arbitraje_comercial_conveciones_guia_interpretacio n_convencion_ny.pdf

17 Traducción libre de: At first sight, it is difficult to see a distinction between the terms 'inoperative' and 'incapable of being performed'. However, an arbitration clause is inoperative where it has 'ceased to have legal effect' as a result, for example, of a failure by the parties to comply with a time limit, or where the parties have repudiated, or by their conduct impliedly revoked, the arbitration agreement.

REDFERN y HUNTER. "International Arbitration" (sexta edición). Kluwer Law International; Oxford University Press, 2015, p. 103.

18 INTERNATIONAL COUNCIL OF COMMERCIAL ARBITRATION. Ibid. p. 53-54 
arbitrales patológicos" o "clauses pathologiques" se utiliza para aquellos pactos arbitrales que por defectos, imperfecciones o deficiencias en la redacción impiden un normal desarrollo del arbitraje, como bien señala Valença cuando cita a Eisemann: "es patológico el convenio arbitral cuya redacción no permite la realización de cualquiera de sus efectos." 19 .

Así, Eisemann señala que existen cuatro elementos esenciales de una cláusula arbitral, estos son:

(i) Producir efectos obligatorios sobre las partes.

(ii) Excluir la intervención de las cortes nacionales sobre la resolución de disputas (al menos sobre los temas contenidos en el laudo).

(iii) Dar poderes a los árbitros para resolver las disputas entre las partes.

(iv) Permitir el establecimiento de una conducción del procedimiento en las mejores condiciones de eficiencia y rapidez para que se dicte un laudo que sea ejecutable judicialmente.

Entonces, la cláusula arbitral será patológica en la medida en que se vaya alejando de los elementos planteados, es decir, siempre que no pueda desplegar todos sus efectos jurídicos; cuando sea inválida o inaplicable ${ }^{20}$.

Es así que autores como César Rivera sostienen que pueden surgir patologías, tanto en convenios arbitrales de reducido contenido, como en aquéllos de contenido amplio y que incluyen una regulación más pormenorizada del arbitraje. Además, señala que puede no solo resultar del convenio arbitral en sí mismo, sino de la falta de coordinación de varios convenios arbitrales que afectan a diversas relaciones jurídicas existentes entre las partes estrechamente vinculadas entre sí (por ejemplo, un pacto de socios y los estatutos de la sociedad o un contrato principal y un acuerdo de garantía para cumplimiento). ${ }^{21}$

\section{iv. La excepción de convenio arbitral en la Ley Peruana de Arbitraje}

19 VALENÇA FILHO, “Clássicos da Arbitragem: Nota Introdutória ao Artigo "La clause d'arbitrage pathologique", de Frédéric Eisemann', en João Bosco Lee y Daniel de Andrade Levy (eds), Revista Brasileira de Arbitragem. Comitê Brasileiro de Arbitragem CBAr \& IOB; Kluwer Law International, 2017, Volume XIV Issue 53. pp. 158 - 162.

20 EISEMANN, Frederic. "La clause d'arbitrage pathologique". En Commercial Arbitration Essays in Memoriam Eugenio Minoli. Torino, Unione Tipografico-editrice Torinese, 1974.

21 COLLANTES GONZÁLEZ, Jorge Luis; BAIZEAU, Domitille; CREMADES, Anne-Carole; DE LA VEGA JUSTRIBÓ, Bárbara; GALLEGO, Jaime; MAS TALADRIZ Jorge y ZAMBRANA TÉVAR, Nicolás. “Diccionario terminológico del arbitraje nacional e internacional (Comercial y de Inversiones)". 2011, p. 352. 
El artículo 16 de la Ley establece lo siguiente:

“Artículo 16.- Excepción de convenio arbitral.

1. Si se interpone una demanda judicial respecto de una materia sometida a arbitraje, esta circunstancia podrá ser invocada como excepción de convenio arbitral aun cuando no se hubiera iniciado el arbitraje.

2. La excepción se plantea dentro del plazo previsto en cada vía procesal, acreditando la existencia del convenio arbitral y, de ser el caso, el inicio del arbitraje.

3. La excepción de convenio arbitral, sea que se formule antes o después de iniciado el arbitraje, será amparada por el solo mérito de la existencia del convenio arbitral, salvo en el primer caso, cuando el convenio fuese manifiestamente nulo.

4. En el arbitraje internacional, si no estuviera iniciado el arbitraje, la autoridad judicial sólo denegará la excepción cuando compruebe que el convenio arbitral es manifiestamente nulo de acuerdo con las normas jurídicas elegidas por las partes para regir el convenio arbitral o las normas jurídicas aplicables al fondo de la controversia. No obstante, si el convenio arbitral cumple los requisitos establecidos por el derecho peruano, no podrá denegarse la excepción. Si estuviera iniciado el arbitraje, la autoridad judicial sólo denegará la excepción cuando compruebe que la materia viola manifiestamente el orden público internacional.

5. Las actuaciones arbitrales podrán iniciarse o proseguir, pudiendo incluso, a discreción del tribunal arbitral, dictarse el laudo, aun cuando se encuentre en trámite la excepción de convenio arbitral" (el énfasis es nuestro).

La Ley Peruana de Arbitraje se ha colocado en distintos supuestos. En primer lugar, hace una introducción a cuándo se puede recurrir a la excepción de convenio arbitral; luego, dispone que quien interponga la excepción se debe remitir a cada vía procedimental específica para determinar los plazos. Asimismo, agrega que se debe acreditar la existencia del convenio arbitral y, de ser el caso, el inicio del arbitraje.

En cuanto al fondo, distingue el arbitraje nacional del arbitraje internacional; sin embargo, en ambos casos cuando no se hubiera iniciado el arbitraje, la única causal para no remitir a las partes al arbitraje es que el convenio arbitral sea manifiestamente nulo. Si el arbitraje estuviera iniciado, en el primer caso, el juez no podría denegar la excepción; mientras que en un arbitraje internacional la única causal que lo habilita a declarar infundada la excepción es que la materia que se pretenda someter a arbitraje viole manifiestamente el orden público internacional. 
A simple vista la regulación de la excepción de convenio arbitral en el Perú habilita al juez a revisar solamente la validez del convenio arbitral; sin embargo, no todos comparten dicha posición. Así, Adrián Simons sostiene lo siguiente:

"Ahora bien en lo que respecta a la posición asumida por el legislador peruano, en el inciso 3) del artículo $16^{\circ}$ de la L.A. se establece que la excepción deberá ser amparada por el solo mérito de la existencia del convenio arbitral, salvo cuando el convenio fuese manifiestamente nulo. Ello quiere decir que el juez estaría prohibido de realizar un análisis exhaustivo de todos los elementos vinculados al convenio arbitral, con la salvedad hecha respecto a la nulidad del convenio.

Sin embargo consideramos que la referida norma nacional debe ser interpretada de manera sistemática con los tratados internacionales de los cuales el Perú es signatario. [...] Por ello integrando lo dispuesto por el inciso 3) del artículo 16 de la LA con la norma II.3 de la Convención de Nueva York, es posible que el juez verifique tres condiciones mínimas para la procedencia de la excepción de convenio arbitral: existencia, validez y vigencia del convenio arbitral" 22 .

Nos resulta imposible adscribirnos a la posición arriba planteada. En primer lugar, debemos tener en cuenta que el inciso 3) de la Ley Peruana de Arbitraje regula la excepción de incompetencia para un arbitraje nacional; sin embargo, como ya hemos expresado líneas arriba, la Convención de Nueva York no aplica en el caso de arbitrajes nacionales, sino solamente en los arbitrajes internacionales. Por lo tanto, en ese caso, la comparación debería ser realizada con el inciso 4) de la norma peruana.

En segundo lugar, no resulta necesario integrar las normas ahí donde no existe un vacío normativo. El legislador peruano no se olvidó de regular los supuestos de ineficacia e inaplicabilidad en la excepción de convenio arbitral. De hecho, la regulación de nuestra excepción tiene su antecedente en la excepción de convenio arbitral del Nuevo Código de Procedimientos Civiles de Francia de 1981. El artículo 1458 establecía lo siguiente:

Artículo 1458

22 SIMONS, Adrián en SOTO, Carlos y BULLARD, Alfredo. “Comentarios a la ley peruana de arbitraje". Lima: Instituto Peruano de Arbitraje Comercial y Arbitraje de Inversiones, 2011, p. 264-265. 
"Cuando una controversia que esté conociendo un tribunal arbitral en virtud de un convenio arbitral, se someta a un tribunal estatal, deberá éste declararse incompetente.

Si el tribunal arbitral no estuviera aún conociendo de la controversia, el tribunal estatal deberá igualmente declararse incompetente, a menos que el convenio arbitral fuera manifiestamente nulo".

Al respecto, Roger Rubio señala que "el Competence-Competence francés es así una regla de prioridad que implica que los tribunales estatales deben remitir a las partes al arbitraje, luego de un examen prima facie de la existencia y validez del convenio arbitral, cuando un tribunal arbitral está conociendo del caso; debiendo, luego del laudo, revisar la jurisdicción de los árbitros. Cuando el tribunal arbitral todavía no estuviera conociendo del caso, los tribunales estatales solo retendrán jurisdicción, cuando el convenio arbitral $\underline{\text { fuera manifiestamente nulo" }}^{23}$ (el énfasis es nuestro).

En conclusión, el legislador peruano ha establecido una regulación distinta a la contenida en el artículo II.3 de la Convención de Nueva York, pues en la propia norma ha estipulado que el análisis del juez debe ser prima facie, es decir, debe analizar el convenio arbitral de manera superficial. Ello implica que si el convenio arbitral tiene al menos una apariencia de validez, el juez debe remitir a las partes a arbitraje.

\section{iv. Comparación entre las excepciones de convenio arbitral}

Como hemos señalado, la excepción de la Convención de Nueva York difiere de la regulada en la Ley Peruana de Arbitraje. La primera, al menos en lo expresamente redactado, permite un análisis más riguroso de la nulidad, eficacia y aplicabilidad; mientras que la Ley Peruana solamente permite que el juez realice un análisis prima facie sobre la nulidad del convenio arbitral. Ello quiere decir, a nuestro juicio, que la excepción de convenio arbitral de la Ley Peruana es más favorable que la de la Convención de Nueva York.

Una visión que sustenta nuestra posición es la de Magdaleno, quien sostiene que "al menos en España, [...] se puede afirmar que la actuación de los Juzgados en torno a la revisión de la cláusula arbitral escapa de un mero control prima facie, para centrarse en un estudio detallado del contenido del pacto. Precisamente este control pleno de validez del convenio arbitral es el expresamente recogido tanto en el artículo II.3 del Convenio de Nueva York

23 RUBIO, Roger. “El principio Competence-Competence en la nueva Ley Peruana de Arbitraje". En Lima Arbitration. N 4, 2011, p. 103. 
como el artículo 8.1 de la Ley Modelo UNCITRAL. A la vista de dicho contenido, y teniendo en cuenta que en España la Ley de Arbitraje sigue el modelo inspirado por la Ley Modelo UNCITRAL, todo parece indicar que el criterio a seguir es el de la cognición plena, si bien el anteproyecto de ley de arbitraje apunta la idea de una cognición limitada, al utilizar el término «manifiestamente nulo»" 24 .

Asimismo, en este punto resulta muy ilustrativo Gary Born pues señala la razón por la que ambas regulaciones son distintas. El autor explica que en la redacción de la excepción de convenio arbitral en la Ley Modelo UNCITRAL que es igual a la de la Convención de Nueva York - se discutió la inclusión de la expresión "manifiestamente nulo". Así, sostiene que:

"La historia de la redacción de la Ley Modelo también apuntó en la dirección de permitir una consideración judicial interlocutoria total de al menos algunas objeciones jurisdiccionales. La mayor evidencia es el hecho que los redactores de la Ley rechazan la propuesta que habría prevista expresamente, en lo que ahora es el Artículo 8.1, para que los tribunales nacionales remitan las controversias a arbitraje, salvo cuando un tribunal 'considere que el acuerdo es manifiestamente nulo'. El rechazo de este lenguaje fue particularmente significativo dado que era precisamente paralelo al texto del nuevo Código de Procedimiento Civil francés de 1981, recientemente adoptado, que preveía una revisión judicial prima facie solamente. El rechazo de una propuesta que habría replicado este enfoque confirma el significado natural del lenguaje del Artículo 8.1 que permite una revisión judicial completa, no prima facie" 25 .

Por otro lado, es interesante analizar de qué manera las Cortes francesas han interpretado la excepción de convenio arbitral regulada en su sistema jurídico. Así la Corte de Casaciones establece lo siguiente:

24 MAGDALENO, Antonia. “Asistencia judicial (en el ámbito arbitral)”. En Diccionario terminológico del arbitraje nacional e internacional (Comercial y de Inversiones), 2011, pp. 284-286.

25 Traducción libre de: "The drafting history of the Model Law also points in the direction of permitting full interlocutory judicial consideration of at least some jurisdictional objections. The most direct evidence is the fact that the Law's drafters rejected a proposal that would have expressly provided, in what is now Article 8(1), for national courts to refer claims to arbitration, save where a court "finds that the agreement is manifestly null and void." The rejection of this language was particularly significant given that it was precisely parallel to the text of the then recently-adopted 1981 French New Code of Civil Procedure, which provided for only prima facie judicial review. Rejection of a proposal that would have replicated this approach confirms the natural meaning of the language of Article 8(1) as permitting full, not prima facie, judicial review".

BORN, Gary. "International Commercial Arbitration". Segunda edición. Kluwer Law International; Kluwer Law International, 2014, p. 1082. 
"Mientras que la Convención de Nueva York del 10 de junio de 1958 se reserva la aplicación de una ley interna más favorable para el reconocimiento de la validez del acuerdo de arbitraje, que es el caso del derecho francés; que, de acuerdo con este derecho, la combinación de los principios de validez de la cláusula de arbitraje internacional y Competence-competence prohíbe que el juez del Estado se pronuncie sobre la existencia, validez y alcance del convenio arbitral antes que el árbitro no se pronuncie sobre este punto, a menos que la cláusula sea manifiestamente nula o inaplicable" 26 .

Resulta interesante ver cómo las Cortes francesas han considerado que el término "manifiestamente nulo" permite que el juez analice tanto la nulidad como aplicabilidad del convenio arbitral. Sin embargo, ello no siempre fue así. De esa manera lo reportan Poudret y Besson "al contrario de una antigua jurisprudencia, los tribunales franceses reconocen hoy que pueden examinar no solo si el acuerdo de arbitraje es manifiestamente nulo, sino también si es manifiestamente inaplicable"27. Consideramos que por esa razón, y para hacer más previsible la excepción, en el 2011 el legislador incluyó el término "inaplicable" en el artículo que regula la excepción de convenio arbitral.

Aunque en el Perú no se ha desarrollado jurisprudencialmente si el término "manifiestamente nulo" incluye el supuesto de inaplicabilidad del convenio arbitral, si esto fuese así, la Ley Peruana culmina la discusión sobre si el análisis debe ser exhaustivo o prima facie $^{28}$.

En conclusión, a nuestro juicio, la excepción de convenio arbitral regulada en el artículo 16 de la Ley Peruana de Arbitraje, es más favorable que la regulada en el artículo II.3 de la Convención de Nueva York, pues dispone un análisis prima facie.

\section{IV. ¿Qué excepción debe ser aplicada?}

La Convención de Nueva York regula en su artículo VII.1 lo siguiente: "las disposiciones de la presente Convención no afectarán la validez de los

26 Sentencia de la Corte de Casaciones del 21 de noviembre de 2016. Groupama Transportes S.A. c. MS Regine Hans y Klaus Heinrich KG.

27 Traducción libre de: "Contrary to a former case law, French courts recognise today that they can examine not only whether the arbitration agreement is manifestly null. but also whether it is manifestly inapplicable".

POUDRET, Jean Francois y BESSON, Sebastien. "Comparative Law of International Arbitration". Editorial Thompson, 2007, p.436.

28 En nuestra opinión, si es posible considerar que el término "manifiestamente nulo" puede incluir supuestos de inaplicabilidad puesto que las Cortes al analizar la excepción no podrían remitir a las partes ante un tribunal arbitral si el convenio fuera manifiestamente inaplicable. 
acuerdos multilaterales o bilaterales relativos al reconocimiento y la ejecución de las sentencias arbitrales concertados por los Estados Contratantes ni privarán a ninguna de las partes interesadas de cualquier derecho que pudiera tener a hacer valer una sentencia arbitral en la forma y medida admitidas por la legislación o los tratados del país donde dicha sentencia se invoque".

Al respecto, el profesor Van den Berg señala que "otro aspecto de la aplicabilidad de la Convención es que la Convención no aplica exclusivamente una vez que un convenio o un acuerdo cae bajo ella. De acuerdo con el artículo VII.1 la ejecución de un laudo arbitral podría también basarse en otro tratado multilateral o bilateral, o ley doméstica relacionada con la ejecución de laudos arbitrales extranjeros, si dicho fundamento es más favorable que la Convención. A pesar que el texto del artículo VII.1 no se refiere a la ejecución de convenios arbitrales, la disposición puede ser considerada que aplica a esta acción" 29 .

En vista a que en el acápite anterior señalamos que la excepción de convenio arbitral de la Ley Peruana de Arbitraje es más favorable, corresponde que el juez resuelva la excepción sobre la base de ella y haga un análisis prima facie del convenio arbitral. Sin perjuicio de lo anterior, debemos tener en cuenta que los jueces prefieren resolver sus causas sobre la base de tratados internacionales antes que las leyes nacionales, por lo que será más efectivo sustentar la excepción de convenio arbitral en ambos instrumentos normativos.

\section{Conclusiones}

Cuando una persona pacta un convenio arbitral tiene la obligación de no recurrir al Poder Judicial para que este resuelva sus controversias. Por ello, con la finalidad de salvaguardar el respeto a dicha obligación, se ha regulado la excepción de convenio arbitral.

En virtud de la Convención de Nueva York se remite a las partes a arbitraje siempre que el convenio arbitral no sea nulo, ineficaz o inaplicable. En el caso

29 Traducción libre de: "Another aspect of the Convention's applicability is that the Convention is not exclusively applicable once an agreement or award falls under it. According to Article VII(1) the enforcement of an arbitral award may also be based on other multilateral or bilateral treaties, or domestic law concerning the enforcement of foreign arbitral awards, if such basis is more favourable than the Convention.

Although the text of Article VII(1) does not refer to the enforcement of the arbitration agreement, the provision can be deemed to apply to this action as well".

VAN DEN BERG, J. Albert. “The New York Arbitration Convention of 1958". Kluwer International Arbitration, 1989, pp. 385-386. 
de la Ley Peruana de Arbitraje, solo basta que el convenio arbitral no sea manifiestamente nulo -es decir, si a primera vista, el convenio es válido, se remitirá a las partes a arbitraje-. No obstante, no es ajeno que ciertas Cortes analicen la aplicabilidad del convenio bajo esa misma expresión, pero siempre bajo un estándar prima facie. Por lo tanto, consideramos que la excepción de la Ley Peruana es más favorable al arbitraje.

Si bien es cierto, la excepción de convenio arbitral de la Ley Peruana de Arbitraje y la regulada en la Convención de Nueva York no aplican siempre a los mismos supuestos, en los casos donde concurra su aplicación, el juez deberá resolver la excepción sustentando su decisión en la Ley Peruana. Ello, en virtud al principio de máxima eficacia regulado en el artículo VII.1 de la Convención de Nueva York.

A pesar de lo señalado, al plantear una excepción de convenio arbitral siempre se debe tener en consideración la importancia de la Convención de Nueva York y la persuasión que puede generar en los jueces. Por lo tanto, consideramos recomendable sustentar la excepción tanto en la Ley Peruana como en la Convención de Nueva York. 